Pacific Journal of Mathematics

METRIC COMPONENTS OF CONTINUOUS IMAGES OF 


\title{
METRIC COMPONENTS OF CONTINUOUS IMAGES OF ORDERED COMPACTA
}

\author{
Joseph N. Simone
}

In this paper the metric component of each point of a Hausdorff space is defined. Several properties of the metric components of continuous images of ordered compacta are then established.

A compactum is a compact Hausdorff space and a continuum is a connected compactum. Any Hausdorff space which can be obtained as a continuous image of an ordered compactum will be called an IOK. Let $X$ be a Hausdorff space. Define the relation $\sim$ on $X$ by $x \sim y$ if and only if there exists a metric continuum in $X$ containing $x$ and $y$. For each $x$ in $X$, let $M_{x}=\{y \in X \mid x \sim y\} . \quad M_{x}$ is called the metric component of $x$. In this paper we will study the properties of metric components of connected IOK's. Our first theorem follows immediately from the above definitions.

THEOREM 1. For each Hausdorff space $X, \sim$ is an equivalence relation on $X$ and $M_{x}$ is connected for each $x$ in $X$.

In general, the metric components of connected IOK's do not have to be compact. This can be seen by considering the "long line." However, under the hypotheses of the next theorem, we obtain the desired result.

THEOREM 2. If $X$ is a first countable IOK, then $M_{x}$ is a continuum for each $x$ in $X$.

Proof. Let $x \in X$. We will show that $M_{x}$ is closed in $X$. Let $y$ be a limit point of $M_{x}$ and let $\left\{U_{n} \mid n \in N\right\}$ where $N$ denotes the set of natural numbers, be a countable base at $y$. For each $n$, let $x_{n} \in U_{n} \cap M_{x}$ and let $K_{n}$ be a metric subcontinuum of $X$ containing $x$ and $x_{n}$. Let $K=\mathrm{Cl}_{n} K_{n}$. Clearly, $K$ is a continuum and $x, y \in$ $K$. Since each $K_{n}$ is separable, it follows immediately that $K$ is separable. However, $K$ is a closed subset of $X$, and therefore $K$ is a separable connected IOK. It follows that $K$ is metrizable [8]. Thus, $y \in M_{x}$ and hence $M_{x}$ is closed.

A space $X$ is paraseparable (Suslinian) if each collection of disjoint nonempty open sets (nondegenerate continua) in $X$ is countable. Every Suslinian continuum is paraseparable [7] and every parase- 
parable IOK is first countable [5]. Thus, every Suslinian connected IOK is first countable. If $S$ is a net whose domain is the directed set $D$, then we will use the notation $\left\{S_{\alpha^{\prime}} \alpha \in D\right\}$ for $S$. When dealing with sequences, $N$ will always denote the set of natural numbers. If $X$ is a space and $A \subseteq B \cong X$, then we will use the notation $\partial B$ to denote the boundary of $B$ in $X$ and the notation $\partial_{B} A$ to denote the boundary of $A$ in the subspace $B$.

The following theorem is due to A. J. Ward ([9] and [4]).

THEOREM 3. If $X$ is an IOK and $\left\{F_{n}, n \in N\right\}$ is a sequence of disjoint closed subsets of $X$, then $\lim \sup F_{n}$ is separable.

We give a proof for the case when $X$ is paraseparable, which is all that we require.

Proof. Suppose that $X$ is a paraseparable IOK. Then the boundary of every open subset of $X$ is separable ([5] and [3]). Now, each $F_{n}$ is closed, and therefore $\partial\left(X-F_{n}\right)$ is separable. Since each $F_{n}$ is closed, $\partial F_{n}=\partial\left(X-F_{n}\right)$, and therefore $\partial F_{n}$ is separable for every $n$. Hence $\bigcup_{n} \partial F_{n}$ is separable and therefore $\mathrm{Cl}_{n} \partial F_{n}$ is separable. Let $F=\mathrm{Cl}_{n} F_{n}$. Since $\left\{F_{n}, n \in N\right\}$ is a sequence of disjoint closed sets, we have that

$$
\mathrm{Cl} \bigcup_{n} F_{n}=\bigcup_{n} F_{n} \cup \lim \sup F_{n} .
$$

Now, $F$ is a closed subset of $X$ and hence $F$ is a IOK. Thus, $F$ is paraseparable, and therefore each $F$-open set is an $F_{\sigma}$ in $F$ [5]. Let

$$
M=\bigcup_{n} F_{n}-\lim \sup F_{n} .
$$

Now, $F-M=\lim \sup F_{n}$, and hence $M$ is $F$-open. Thus, $M$ is an $F_{\sigma}$ in $F$, and therefore $\partial_{F} M$ is separable [3]. Furthermore, since $F$ is closed, $M \subseteq F$, and $M$ is $F$-open, it follows that $\partial_{F} M=\bar{M} \cap(F-M)$.

Let

$$
S=\mathrm{Cl} \bigcup_{n} \partial F_{n} \cup \partial_{F} M .
$$

Since $S$ is the union of two closed separable subspaces of $X, S$ is a separable IOK. We claim that $\lim \sup F_{n} \cong S$. Suppose that $x \in$ $\lim \sup F_{n}-\partial_{F} M$. Then $x \notin \bar{M}$. Let $V$ be an open set such that $x \in V \cong X-\bar{M}$. There exists an $n_{0}$ such that $V \cap F_{n_{0}} \neq \varnothing$. Since $V \cap\left(\bigcup_{n} F_{n}-\lim \sup F_{n}\right)=\varnothing, V \cap\left(F_{n_{0}}-\lim \sup F_{n}\right)=\varnothing$, and hence

$$
V \cap F_{n_{0}} \subseteq \lim \sup F_{n} \subseteq \mathrm{Cl}\left(X-F_{n_{0}}\right) \text {. }
$$


Therefore

$$
V \cap\left(F_{n_{0}} \cap \mathrm{Cl}\left(X-F_{n_{0}}\right)\right)=V \cap \partial F_{n_{0}} \neq \varnothing .
$$

It follows that $V \cap \bigcup_{n} \partial F_{n} \neq \varnothing$, and hence $x \in \mathrm{Cl} \bigcup_{n} \partial F_{n}$. Therefore $\lim \sup F_{n} \leqq S$. Since every closed subset of a separable IOK is separable [5], it follows that $\lim \sup F_{n}$ is separable.

THEOREM 4. If $X$ is a Suslinian connected IOK, and $Y=$ $\left\{M_{x} \mid x \in X\right\}$, then $Y$ is an upper semi-continuous decomposition of $X$.

Proof. By Theorem 2, each $M_{x}$ is a continuum. Thus, $Y$ is certainly a decomposition of $X$. Let $H \in Y$ and let $U$ be an open set such that $H \subseteq U$. Now, since $X$ is Suslinian and the elements of $Y$ are disjoint continua, $Y$ has only countably many nondegenerate members. Let $\mathscr{G}$ denote the set of all elements $M$ of $Y$ such that $M \cap U \neq \varnothing$ and $M \not \subset U$. Since each element of $\mathscr{G}$ is nondegenerate, $\mathscr{G}$ is countable. Let $K=\mathrm{Cl} \cup \mathscr{G}$. We claim that $K \cap H=\varnothing$. Suppose $x \in K \cap H$. Since each element of $\mathscr{G}$ is disjoint from $H$, it follows that $\mathscr{G}$ is infinite. Let $\mathscr{G}=\left\{K_{n} \mid n \in N\right\}$. Since $\left\{K_{n}, n \in N\right\}$ is a sequence of disjoint closed sets,

$$
\mathrm{Cl} \bigcup_{n} K_{n}=\bigcup_{n} K_{n} \cup \lim \sup K_{n} \text {. }
$$

Since $H \cap K_{n}=\varnothing$ for each $n$, it follows that $x \in H \cap \lim \sup K_{n}$. Let $\left\{U_{n} \mid n \in N\right\}$ be a monotone decreasing countable base at $x$. Clearly, there exists a subsequence $\left\{K_{n_{i}}, i \in N\right\}$ of $\left\{K_{n}, n \in N\right\}$ such that $K_{n_{i}} \cap$ $U_{i} \neq \varnothing$ for all $i$. It follows that $x \in \lim \inf K_{n_{i}}$. Thus, $\lim \sup K_{n_{i}}$ is a continuum. By Theorem $3, \lim \sup K_{n_{i}}$ is separable and therefore $\lim \sup K_{n_{i}}$ is a metrizable continuum [8]. Now, for each $K_{n_{i}}, K_{n_{i}} \not \subset$ $U$, so that $K_{n_{i}} \cap(X-U) \neq \varnothing$. Since $X-U$ is compact, there exists a $y$ in $X-U$ such that $y \in \lim \sup K_{n_{i}}$. Thus, $\lim \sup K_{n_{i}}$ is a metric continuum containing $x$ and $y$. However, this is impossible since $H$ is the metric component of $x, H \subseteq U$, and $y \notin U$. It follows that $K \cap H=\varnothing$. Let $V=U-K$. Then $V$ is an open set, and, clearly, $H \leqq V \subseteq U$. Let $L \in Y$ such that $L \cap V \neq \varnothing$. Then, $L \cap U \neq \varnothing$, and since $V=U-\mathrm{Cl}_{n} K_{n}$, it follows that $L \cong U$. Thus, $Y$ is upper semi-continuous.

Whenever $\{A \mid A \in Y\}$ is a decomposition of $X$, it is to be assumed that $Y$ is given the quotient topology derived from the topology of $X$, and that $p$ denotes the natural map from $X$ onto $Y$ given by $p(x)=A$ where $x \in A \in Y$.

THEOREM 5. Let $X$ be a Suslinian connected IOK and let $Y=$ 
$\left\{M_{x} \mid x \in X\right\}$. If $K$ is a subcontinuum of $Y$, then $p^{-1}(K)$ is a subcontinuum of $X$.

Proof. Let $K$ be a subcontinuum of $Y$, and let $K^{*}=p^{-1}(K)$. Since $Y$ is upper semi-continuous, $p$ is continuous and closed. Thus, $K^{*}$ is a compact subset of $X$. Suppose that $K^{*}$ is not connected. Then $K^{*}$ is the union of two disjoint closed subsets $A^{*}$ and $B^{*}$ of $X$. Let $A=p\left(A^{*}\right)$ and $B=p\left(B^{*}\right)$. Then $A$ and $B$ are closed subsets of $Y$ and $A \cup B=K$. Since $K$ is connected we must have that $A \cap B \neq \varnothing$. Let $M_{x} \in A \cap B$. Then $M_{x} \in p\left(A^{*}\right) \cap p\left(B^{*}\right)$, and hence there exist an $a$ in $A^{*}$ and $a b$ in $B^{*}$ such that $p(a)=p(b)=M_{x}$. Thus, $M_{x} \subseteq K^{*}=A^{*} \cup B^{*}$. But $M_{x} \cap A^{*} \neq \varnothing$ and $M_{x} \cap B^{*} \neq \varnothing$, which contradicts the fact that $M_{x}$ is connected. Therefore $K^{*}$ is a continuum.

THEOREM 6. If $X$ is a Suslinian connected IOK, then $M_{x}$ is metrizable for each $x$ in $X$.

Proof. Let $x \in X$. If $M_{x}=\{x\}$, then, clearly, $M_{x}$ is metrizable. So suppose that $M_{x}$ is nondegenerate. Let $\mathscr{S}$ denote the set of all collections of disjoint nondegenerate metric continua contained in $M_{x}$. Clearly, $\mathscr{S} \neq \varnothing$. By Zorn's Lemma it follows immediately that $\mathscr{S}$ has a maximal element $\mathscr{M}$. Since $X$ is Suslinian, $\mathscr{M}$ is countable. Let $\mathscr{M}=\left\{M_{n} \mid n \in N^{\prime}\right\}$, where $N^{\prime}$ is some subset of $N$.

Now, since each $M_{n}$ is a metric continuum, each $M_{n}$ has a countable dense subset $D_{n}$. Let $D=\bigcup_{n} D_{n}$. Then $D$ is countable. We claim that $D$ is dense in $M_{x}$. Suppose that $y \in M_{x}-D$. By definition there exists a metric continuum $K$ such that $y \in K \subseteq M_{x}$ and $K \cap$ $D \neq \varnothing$. Let $U$ be any open set containing $y$ and let $V$ be an open set such that $y \in V, \bar{V} \subseteq U$ and $K \cap(X-V) \neq \varnothing$. Then $K \cap V$ is a proper $K$-open set. Let $C$ be the component of $y$ in $K \cap V$. Then $\bar{C} \cap \partial_{K}(K \cap V) \neq \varnothing[2]$, and hence $\bar{C}$ is a nondegenerate subcontinuum of $M_{x}$. Furthermore, $\bar{C} \leqq K$ and therefore, $\bar{C}$ is metric. Since $\mathscr{M}$ is maximal, $\bar{C} \cap M_{n} \neq \varnothing$ for some $n$. However, $\bar{C} \subseteq U$, and hence $U \cap M_{n} \neq \varnothing$. But then $U \cap D \neq \varnothing$, so $y \in \bar{D}$. Thus, $D$ is dense in $M_{x}$. Since $M_{x}$ is a connected IOK, it follows that $M_{x}$ is metrizable [8].

A continuum $X$ is netlike if each pair of points in $X$ can be separated by a finite set. The following theorem is proved in [7].

THEOREM 7. If $X$ is a paraseparable continuum containing no nondegenerate metric subcontinuum, then $X$ is netlike if and only if it is an IOK. 
THEOREM 8. If $X$ is a Suslinian connected IOK, and $Y=$ $\left\{M_{x} \mid x \in X\right\}$, then $Y$ is a netlike continuum.

Proof. Let $Y=\left\{M_{x} \mid x \in X\right\}$. Then $Y$ is an upper semi-continuous decomposition of $X$ and $Y$ is a continuum. Let $p$ be the natural map from $X$ onto $Y$. Since $Y$ is upper semi-continuous, $p$ is continuous and closed. It follows from Theorem 5 that $Y$ is Suslinian. Therefore $Y$ is a paraseparable connected IOK.

We claim that $Y$ contains no nondegenerate metric subcontinuum. Suppose that $K$ is a nondegenerate metric subcontinuum of $Y$. Let $H=p^{-1}(K)$. By Theorem $5, H$ is a nondegenerate subcontinuum of $X$. Now, since $X$ is Suslinian, $Y$ has only countably many nondegenerate members. Let $\left\{p\left(x_{n}\right) \mid n \in N^{\prime}\right\}$, where $N^{\prime} \subseteq N$, denote the set of nondegenerate elements of $Y$ contained in $H$. By Theorem 6, each $M_{x_{n}}$ is a metric continuum and hence contains a countable dense set $D_{n}$. Now, $K$ is a metric subcontinuum of $Y$, and hence $K$ has a countable dense subset $E$. Let $F=E-\left\{p\left(x_{n}\right) \mid n \in N^{\prime}\right\}$. Thus, if $M_{x} \in F$, then $M_{x}=\{x\}$. Let $A=p^{-1}(F)$. Then $A$ is a countable subset of $X$. Let $D=A \cup \bigcup_{n} D_{n}$. $D$ is a countable set. We claim that $D$ is dense in $H$. Let $U$ be an $H$-open set. If $U \cap M_{x_{n}} \neq \varnothing$ for some $n$, then $U \cap D_{n} \neq \varnothing$ and therefore $U \cap D \neq \varnothing$. Suppose that $U \cap M_{x_{n}}=\varnothing$ for each $n$. Thus, if $x \in U$, then $p(x)=\{x\}$ and therefore $p^{-1}(p(U))=U$. Hence

$$
p(H-U)=p(H)-p(U)=K-p(U) .
$$

Now, since $H-U$ is a closed set and $p$ in a closed map, $K-p(U)$ is closed. Thus, $p(U)$ is $K$-open. Hence $p(U) \cap E \neq \varnothing$. However, $x \in U$ implies that $p(x)=\{x\}$, and therefore $p(U) \cap F \neq \varnothing$. Let $\{y\} \in p(U) \cap F$. Then $y \in A$ and $y \in p^{-1}(p(U))=U$, so that $U \cap A \neq \varnothing$. Thus, $U \cap D \neq \varnothing$, and therefore $D$ is dense in $H$. Since $H$ is a separable connected IOK, $H$ is metrizable, and therefore $p(H)=K$ is degenerate. Hence $Y$ contains no nondegenerate metric subcontinuum. By Theorem $7, Y$ is netlike.

An hereditarily locally connected continuum is a continuum in which each subcontinuum is locally connected. By combining Theorems 4,6 and 8 we immediately obtain the following result.

THEOREM 9. If $X$ is a Suslinian connected hereditarily locally connected IOK, then there exists an upper semi-continuous decomposition $Y$ of $X$ such that the space $Y$ is a netlike continuum and each element of $Y$ is a Peano space.

The author would like to thank Professor A. J. Ward for his 
comments on this paper, and in particular for his remarks which led to the present form of Theorem 6 .

\section{REFERENCES}

1. Z. Frolík, Concerning topological convergence of sets, Czechoslovak Math. J., 10 (85)(1960), 168-180.

2. J. G. Hocking and G. S. Young, Topology, Addison-Wesley Publishing Co., Reading, 1961.

3. S. Mardešić, Continuous images of ordered compacta and a new dimension which neglects metric subcontinua, Trans. Amer. Math. Soc., 121 (1966), 424-433.

4. - On the Hahn-Mazurkiewicz problem in non-metric spaces, General Topology and its Relation to Modern Analysis and Algebra II (1967), 248-255.

5. S. Mardešić and P. Papić, Continuous images of ordered compacta, Glasnik Mat.Fiz. Astronom., 17 (1962), 3-25.

6. B. J. Pearson, Mapping arcs and dendritic spaces onto netlike continua, Coll. Math., 34 (1975), 39-48.

7. J. N. Simone, Continuous images of ordered compacta and hereditarily locally connected continua, submitted to Fund. Math.

8. L. B. Treybig, Concerning continua which are continuous images of compact ordered spaces, Duke Math. J., 32 (1965), 417-422.

9. A. J. Ward, Some properties of images of ordered compacta, with special reference to topological limits, unpublished.

10. L. E. Ward, Jr., The Hahn-Mazurkiewicz theorem for rim-finite continua, General Topology and Appl., 6 (1976), 183-190.

11. G. T. Whyburn, Analytic topology, Amer. Math. Soc. Colloq. Publ., 28 (1942).

Received April 20, 1976. This paper is part of the author's doctoral dissertation under the direction of Professor B. J. Pearson.

UNIVERSITY OF MISSOURI 


\section{PACIFIC JOURNAL OF MATHEMATICS}

EDITORS

RICHARD ARENS (Managing Editor)

University of California

Los Angeles, California 90024

R. A. Beaumont

University of Washington

Seattle, Washington 98105
J. DUGUNDJI

Department of Mathematics

University of Southern Californı

Los Angeles, California 90007

D. Gilbarg and J. Milgram

Stanford University

Stanford, California 94305

\section{ASSOCIATE EDITORS}

E. F. BECKENBACH

B. H. NeumanN

F. WOLF

K. YoSHIDA

\section{SUPPORTING INSTITUTIONS}

UNIVERSITY OF BRITISH COLUMBIA

CALIFORNIA INSTITUTE OF TECHNOLOGY

UNIVERSITY OF CALIFORNIA

MONTANA STATE UNIVERSITY

UNIVERSITY OF NEVADA

NEW MEXICO STATE UNIVERSITY

OREGON STATE UNIVERSITY

UNIVERSITY OF OREGON

OSAKA UNIVERSITY
UNIVERSITY OF SOUTHERN CALIFORNIA

STANFORD UNIVERSITY

UNIVERSITY OF TOKYO

UNIVERSITY OF UTAH

WASHINGTON STATE UNIVERSITY

UNIVERSITY OF WASHINGTON

AMERICAN MATHEMATICAL SOCIETY

NAVAL WEAPONS CENTER 


\section{Pacific Journal of Mathematics}

Vol. 69 , No. 1

May, 1977

V. V. Anh and P. D. Tuan, On starlikeness and convexity of certain analytic

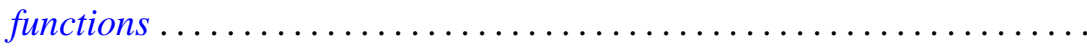

Willard Ellis Baxter and L. A. Casciotti, Rings with involution and the prime

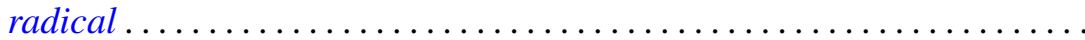

Manuel Phillip Berriozabal, Hon-Fei Lai and Dix Hayes Pettey,

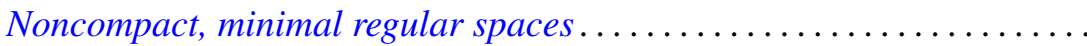

Sun Man Chang, Measures with continuous image law ................

John Benjamin Friedlander, Certain hypotheses concerning

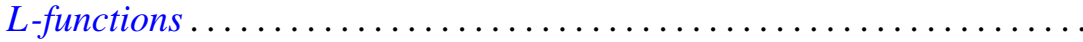

Moshe Goldberg and Ernst Gabor Straus, On characterizations and

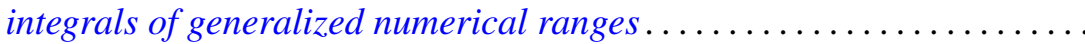

Pierre A. Grillet, On subdirectly irreducible commutative semigroups ...... 55

Robert E. Hartwig and Jiang Luh, On finite regular rings ..............

Roger Hugh Hunter, Fred Richman and Elbert A. Walker, Finite direct sums of cyclic valuated p-groups ........................... 97

Atsushi Inoue, On a class of unbounded operator algebras. III ......... 105

Wells Johnson and Kevin J. Mitchell, Symmetries for sums of the Legendre

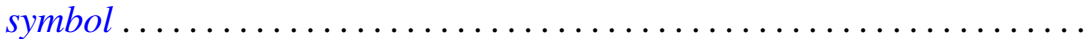

Jimmie Don Lawson, John Robie Liukkonen and Michael William Mislove,

Measure algebras of semilattices with finite breadth

Glenn Richard Luecke, A note on spectral continuity and on spectral properties of essentially $G_{1}$ operators ...............

Takahiko Nakazi, Invariant subspaces of weak-* Dirichlet algebras . .

James William Pendergrass, Calculations of the Schur group ...

Carl Pomerance, On composite $n$ for which $\varphi(n) \mid n-1$. II. . .

Marc Aristide Rieffel and Alfons Van Daele, A bounded operator approach to Tomita-Takesaki theory........................

Daniel Byron Shapiro, Spaces of similarities. IV. $(s, t)$-families ...

Leon M. Simon, Equations of mean curvature type in 2 independent variables.

Joseph Nicholas Simone, Metric components of continuous images of ordered compacta ............................

William Charles Waterhouse, Pairs of symmetric bilinear forms in

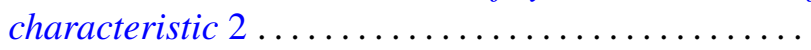

